\title{
Mesenchymal stem cells decrease blood-brain barrier permeability in rats with severe acute pancreatitis
}

\author{
Ronggui Lin ${ }^{1 \dagger}$, Ming Li ${ }^{2 \dagger}$, Meiqin Luo ${ }^{3}$, Tianhong Teng ${ }^{1}$, Yu Pan ${ }^{1}$ and Heguang Huang ${ }^{1 *}$
}

\author{
* Correspondence: \\ Heguanghuang2@163.com \\ ${ }^{\dagger}$ Ronggui Lin and Ming Li \\ contributed equally to this work. \\ ${ }^{1}$ Department of General surgery, \\ Fujian Medical University Union \\ Hospital, 29 Xinquan Road, Fuzhou, \\ Fujian 350001, People's Republic of \\ China \\ Full list of author information is \\ available at the end of the article
}

\begin{abstract}
Background: Impairment of the blood-brain barrier (BBB) could result in secondary cerebral edema and life-threatening pancreatic encephalopathy in patients with severe acute pancreatitis (SAP). Mesenchymal stem cells (MSCs) have been widely adopted in clinical research because of their pleiotropic functions. The aim of this study was to investigate the impact of MSCs on BBB permeability in SAP and the potential mechanisms driving these effects.

Methods: Sprague-Dawley rats were randomly assigned to the control, SAP and SAP+MSCs groups. Pancreatic impairment was assessed. The serum levels of amylase, TNF- $a$ and IL-10, expression levels of claudin-5, Bax, BCl-2 and MMP-9, and the BBB permeability were measured. Endothelial cell apoptosis was evaluated.

Results: SAP rats showed BBB impairment with increased permeability and secondary cerebral edema, which was confirmed using the Evans blue assay and the calculation of the brain dry/wet ratio. Treatment with MSCs decreased the serum levels of amylase and TNF-a, increased the serum levels of IL-10, attenuated the apoptosis of brain microvascular endothelial cells, upregulated claudin-5 expression and downregulated MMP-9 expression. This treatment attenuated the increased BBB permeability in SAP rats.

Conclusions: MSCs attenuated the impairment of the BBB and decreased its permeability, producing protective effects in SAP rats.

Keywords: Severe acute pancreatitis, Blood-brain barrier, Mesenchymal stem cell, Inflammatory response, Endothelial cell
\end{abstract}

\section{Background}

Pancreatic encephalopathy (PE) is a rare and serious complication of severe acute pancreatitis (SAP) presenting with psychogeny, with a high mortality rate of approximately $67 \%$ [1]. The underlying pathogenesis remains to be explored, but the main promising hypothesis suggests that PE development is closely related to a blood-brain barrier (BBB) impairment that causes increased permeability.

The BBB protects the central nervous system from pathogens [2]. It is mainly composed of brain microvascular endothelial cells (BMECs), paracellular junctions, astrocytes, pericytes and the basement membrane [3]. BMECs and paracellular junctions are the structural and functional components of the BBB. Paracellular junctions

(c) The Author(s). 2019 Open Access This article is distributed under the terms of the Creative Commons Attribution 4.0 International License (http://creativecommons.org/licenses/by/4.0/), which permits unrestricted use, distribution, and reproduction in any medium, provided you give appropriate credit to the original author(s) and the source, provide a link to the Creative Commons license, and indicate if changes were made. The Creative Commons Public Domain Dedication waiver (http://creativecommons.org/ publicdomain/zero/1.0/) applies to the data made available in this article, unless otherwise stated. 
include tight, adherens and gap junctions [4-6], and claudin-5 is a critical tight junction protein for maintaining the integrity and stability of the barrier [7].

Matrix metalloproteinase-9 (MMP-9) is a member of the zinc-dependent metalloproteinase family, which is involved in the degradation of extracellular matrix components. It has been reported that MMP-9 causes increased degradation of collagen IV in the vascular basement membrane and a subsequent increase in BBB permeability in diabetic mice following a stroke [8]. MMP-9 is often upregulated by various inflammatory cytokines and mediators in an aggravated inflammatory response following brain injury, leading to an increase in BBB permeability. However, it is unclear whether MMP-9 is upregulated in SAP rats with increased BBB permeability.

Mesenchymal stem cells (MSCs) have been widely applied in clinical research as a result of their superior multipotential differentiation, low immunogenicity and paracrine function. A recent study demonstrated that they exert immunomodulatory effects during the treatment of various inflammatory diseases [9]. Another study showed that they promote tissue repair and have anticancer properties [10]. In our previous study, we demonstrated that MSCs protect the endothelial barrier in the small intestine and kidney in SAP rats $[11,12]$. However, their similar effects on the BBB in SAP rats and the underlying mechanisms remain unclear. In this study, an SAP rat model was established to study the potential mechanisms of impairment of the BBB and the effects of MSCs on this important barrier.

\section{Materials and methods}

\section{Animals}

Germ-free adult male Sprague-Dawley (SD) rats weighing 200-250 g were obtained from Shanghai SLAC Laboratory Animal Co. Ltd. They were allowed to acclimatize for a week in rooms with a 12-h light-dark cycle at a temperature of $20 \pm 2{ }^{\circ} \mathrm{C}$. All animal experimental protocols were approved by the Experimental Animals Committee of Fujian Medical University. All experimental animals received humane care and were treated according to ethical guidelines and standards [13].

The rats $(n=30)$ were randomly divided into 3 groups: control, SAP and SAP+MSCs ( $n=10$ per group). An SAP model was established for the rats in the SAP and $\mathrm{SAP}+\mathrm{MSCs}$ groups. The rats in the SAP+MSCs group received $1 \mathrm{ml}$ of MSCs (approximately $1 \times 10^{6}$ cells $/ \mathrm{ml}$ ) via an injection into the femoral vein after the establishment of the model. All the animals were euthanized after $12 \mathrm{~h}$ for sample collection.

\section{Isolation, culture and identification of MSCs}

MSCs were isolated using the differential adherence method, as described previously [14]. The tibia and femur of a 1-month old SD rat were separated, and the bone marrow cavities were exposed and flushed with Dulbecco's modified Eagle's medium (DMEM; HyClone). The collected DMEM was centrifuged at $300 \times \mathrm{g}$ for $5 \mathrm{~min}$, then resuspended, supplemented with 10\% (w/v) fetal bovine serum (FBS; Gibco) and 1\% penicillin/streptomycin (HyClone). The cells were inoculated in a $25-\mathrm{cm}^{2}$ culture flask at a concentration of $5 \times 10^{7}$ cells $/ \mathrm{ml}$, then incubated at $37{ }^{\circ} \mathrm{C}$ with $5 \% \mathrm{CO}_{2}$. Non-adherent cells were removed by washing the flask with phosphate-buffered saline (PBS; HyClone) three times $24 \mathrm{~h}$ later. The medium was changed every $2-3$ days until the cells reached 
a confluency of $80-90 \%$. The MSCs were purified by dissociation, and the third generation was used for further experiments.

MSCs were identified using flow cytometry analysis (FACS, Beckton Dickinson) by detecting the cell surface markers CD29, CD34, CD45 and CD90. The respective phycoerythrin-conjugated primary antibodies were a mouse/rat anti-CD29 antibody (1 $\mathrm{g}$; eBioscience), mouse anti-CD34 antibody (1 $\mathrm{g}$; Santa Cruz Biotechnology), rat anti-CD45 antibody (0.25 $\mu \mathrm{g}$; eBioscience) and mouse/rat anti-CD90 antibody $(0.06 \mu \mathrm{g}$, eBioscience).

\section{Establishment of an SAP animal model}

Retrograde injection of $5 \%$ sodium taurocholate $(0.1 \mathrm{ml} / 100 \mathrm{~g}$ body weight, Inalco Spa) into the biliopancreatic duct was used to establish an SAP rat model, as described previously $[15,16]$. Before the operation, the rats were allowed to drink water but were fasted for $12 \mathrm{~h}$. Anesthesia was performed via an abdominal cavity injection with $10 \%$ chloral hydrate ( $3 \mathrm{ml} / \mathrm{kg}$ body weight, Bio Basic). A 1 - to 1.5 - $\mathrm{cm}$ incision in the midline of the upper abdomen was used for the laparotomy. A $0.45-\mathrm{mm}$ diameter polyethylene catheter was used for the injection, which was performed at a speed of $0.04 \mathrm{ml} / \mathrm{min}$ using a microinfusion pump that was removed $10 \mathrm{~min}$ later. In the control group, the pancreas and duodenum were maneuvered during the laparotomy without any injection into the biliopancreatic duct. The abdomen was closed with sutures, and after the surgery, the rats were given $4 \mathrm{ml}$ normal saline/100 g body weight every $6 \mathrm{~h}$ via subcutaneous injection at multiple sites on the back.

\section{Histological analysis}

Four micron sections of the harvested body of the pancreas were stained with hematoxylin and eosin (H\&E), then viewed under a light microscope (Carl Zeiss). The pathological changes were evaluated with a pathological scoring system [17].

\section{Serum amylase detection and enzyme-linked immunosorbent assay (ELISA)}

Serum was obtained from the collected blood samples with centrifugation at $3000 \mathrm{x} \mathrm{g}$ at $4{ }^{\circ} \mathrm{C}$ for $10 \mathrm{~min}$. It was stored at $-80^{\circ} \mathrm{C}$ until further analysis. The serum amylase level was measured with an Olympus AV2700 automated clinical biochemistry analyzer. The serum levels of tumor necrosis factor $\alpha$ (TNF- $\alpha$ ) and interleukin-10 (IL-10) were detected with a rat ELISA kit (R\&D Systems) in accordance with the manufacturer's instructions.

\section{Evans blue assay}

We prepared another 3 groups of experimental rats following the same experimental procedures to measure the permeability of the BBB using the Evans blue assay as previously described [18]. Evans blue solution (Sigma) at a concentration of $2 \%$ at $5 \mathrm{ml} / \mathrm{kg}$ body weight was injected into the femoral vein $1 \mathrm{~h}$ before the rats were euthanized. The circulating dye was cleared with a perfusion of cold PBS. The harvested brain tissue was homogenized and incubated in dicarboxamide at $37^{\circ} \mathrm{C}$ for $48 \mathrm{~h}$. After centrifugation at $300 \times \mathrm{g}$ for $5 \mathrm{~min}$, the optical density (OD) of the supernatants was measured 
at $620 \mathrm{~nm}$ absorbance with a SpectraMax M5e Multi-Mode Microplate Reader (Molecular Devices).

\section{Brain dry/wet ratio calculation}

The brain dry/wet ratio was calculated to evaluate the severity of cerebral edema. The collected frontal lobe was weighed before and after drying in an oven at $80^{\circ} \mathrm{C}$ for $48 \mathrm{~h}$. The ratio was calculated as dry weight (g)/wet weight (g).

\section{Quantitative real-time PCR}

Total RNA was extracted from the brain tissue with Trizol reagent (Life Technologies) followed by reverse transcription into cDNA with a Transcriptor First Strand cDNA Synthesis Kit (Roche) in accordance with the manufacturer's instructions. Quantitative real-time PCR was conducted with a StepOnePlus Real-Time PCR System (Applied Biosystems) with Bestar SybrGreen qPCR Mastermix (DBI Bioscience). Glyceraldehyde3-phosphate dehydrogenase (GAPDH) was used as an internal reference. The relative expression of each gene was calculated with the $2^{-\triangle \Delta C T}$ method. The primer sequences included rat GAPDH, 5'-GCGAGATCCCGCTAACATCA-3' and 5'-GGCACCGT TGGATCATAG-3'; claudin-5, 5'-GCACTCTTTGTTACCTTGAC-3' and 5'-GGCA CCGTTGGATCATAG-3'; Bcl-2-associated X (Bax), 5'-CAGACGGCAACTTCAACT3' and 5'- CTTCCAGATGGTGAGTGA - 3'; and B-cell lymphoma 2 (Bcl-2), 5'GCAGAGATGTCCAGTCAG-3'; 5'-ATCCACAGAGCGATGTTG-3' (as indicated in Addtional file 1).

\section{Western blot analysis}

Brain tissue was homogenized on ice, and ice-cold RIPA lysis buffer (Beyotime) containing $1 \mathrm{mM}$ PMSF (Beyotime) was added to each sample. After centrifugation at 14, $000 \times \mathrm{g}$ at $4{ }^{\circ} \mathrm{C}$ for $10 \mathrm{~min}$, the supernatants were collected. The protein concentration was measured with a BCA kit (Beyotime). Approximately $50 \mu \mathrm{g}$ of protein was separated via SDS-PAGE with an appropriate concentration of SDS. The protein was then transferred to nitrocellulose membranes. The membranes were incubated with the following primary antibodies at $4{ }^{\circ} \mathrm{C}$ overnight: mouse anti-claudin-5 (1:500, Invitrogen), mouse anti-Bax (1:1000, Cell Signaling Technology), rabbit anti-Bcl-2 (1:1000, Abcam), rabbit anti-MMP-9 (1:1000, Abcam) and mouse anti- $\beta$-actin (1:1000, Transgen). Then, the membranes were incubated with the appropriate secondary antibodies at $25^{\circ} \mathrm{C}$ for $2 \mathrm{~h}$ : goat anti-mouse IgG-HRP and goat anti-rabbit IgG-HRP antibodies (1:5000, Cell Signaling Technology). After detection with a ChemiDocTM MP imaging system (Bio$\mathrm{Rad})$, the results were analyzed with Image J software version version 1.48.

\section{Immunohistochemistry staining}

Following dewaxing, rehydration and antigen retrieval, the 4- $\mu \mathrm{m}$ sections of the brain tissue were incubated with hydrogen peroxide to block endogenous peroxidase, then blocked with $5 \%$ bovine serum albumin (BSA; Sigma) for $1 \mathrm{~h}$. The sections were incubated with anti-claudin- 5 antibody (1:50, Invitrogen) at $4{ }^{\circ} \mathrm{C}$ overnight and then with secondary antibody $\left(1: 100\right.$; Abcam) for $30 \mathrm{~min}$ at $37^{\circ} \mathrm{C}$. Peroxidase activity was 
visualized with 3-diaminobenzidine (DAB). Then, the slides were stained with hematoxylin, dehydrated with a gradient alcohol, cleared with xylene, and cover slipped.

\section{Terminal deoxynucleotidyl transferase-mediated nick end labelling (TUNEL) assay}

As previously described [19], the sections of brain tissue were incubated with a TUNEL reaction mixture (TUNEL staining kit; Roche) at $37^{\circ} \mathrm{C}$ for $1 \mathrm{~h}$ following dewaxing, rehydration and blocking endogenous peroxidase. After staining with DAPI (1:1000; Sigma), the sections were viewed under a fluorescence microscope (Carl Zeiss), and the TUNEL- and DAPI-positive cells were counted.

\section{Statistical analysis}

Data are presented as the means \pm SD (standard deviation) and were analyzed with the statistical software SPSS 19.0. Differences between the groups were analyzed using oneway analysis of variance (ANOVA) with multiple comparisons. $p<0.05$ was considered statistically significant.

\section{Results}

The culture and identification of rat MSCs

The MSCs adhering to the wall of the culture flask $24 \mathrm{~h}$ after inoculation were purified via dissociation. The third-generation MSCs were spindle-shaped and adherent (Fig. 1a). After harvesting, the MSCs were identified using flow cytometry for specific cell surface markers, including CD29, CD34, CD45 and CD90. Flow cytometry analysis showed that CD29-, CD34-, CD45- and CD90-positive cells respectively accounted for approximately $99.28,0.94,1.44$ and $97.79 \%$ of the cells, which met the requirements for further experiments (Fig. 1b).

\section{MSCs alleviated pancreatic impairment and decreased BBB permeability in SAP rats}

The typical manifestations of SAP, including ascites and scattered saponification spots on the mesenterium and the greater omentum, were observed when the rats were euthanized. The control group did not show pathological changes in the pancreas based on H\&E staining. Pancreatic edema, hemorrhage, necrotic acini and the infiltration of inflammatory cells were observed in the SAP group under a light microscope (Fig. 2a).
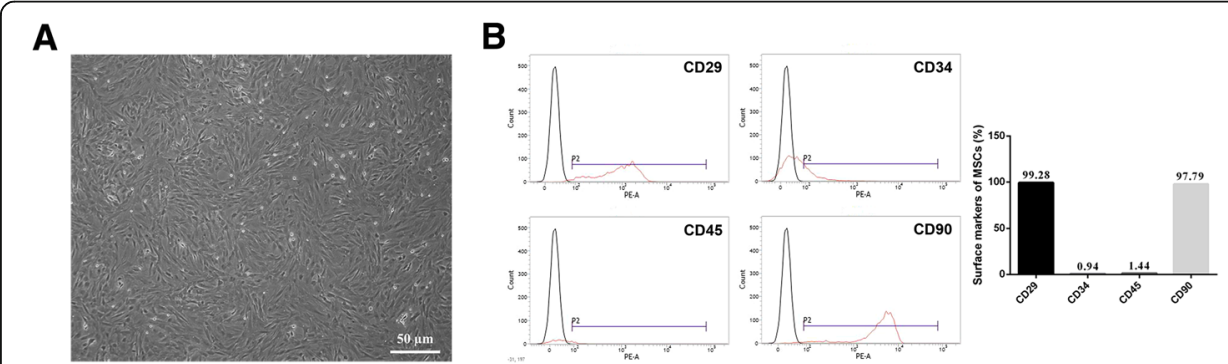

Fig. 1 The culture and identification of rat MSCs. a Morphology of rat MSCs under a light microscope, scale bar represents $50 \mu \mathrm{m}$. b Flow cytometry for the detection of the MSC cell surface markers CD29, CD34, CD45 and CD90 


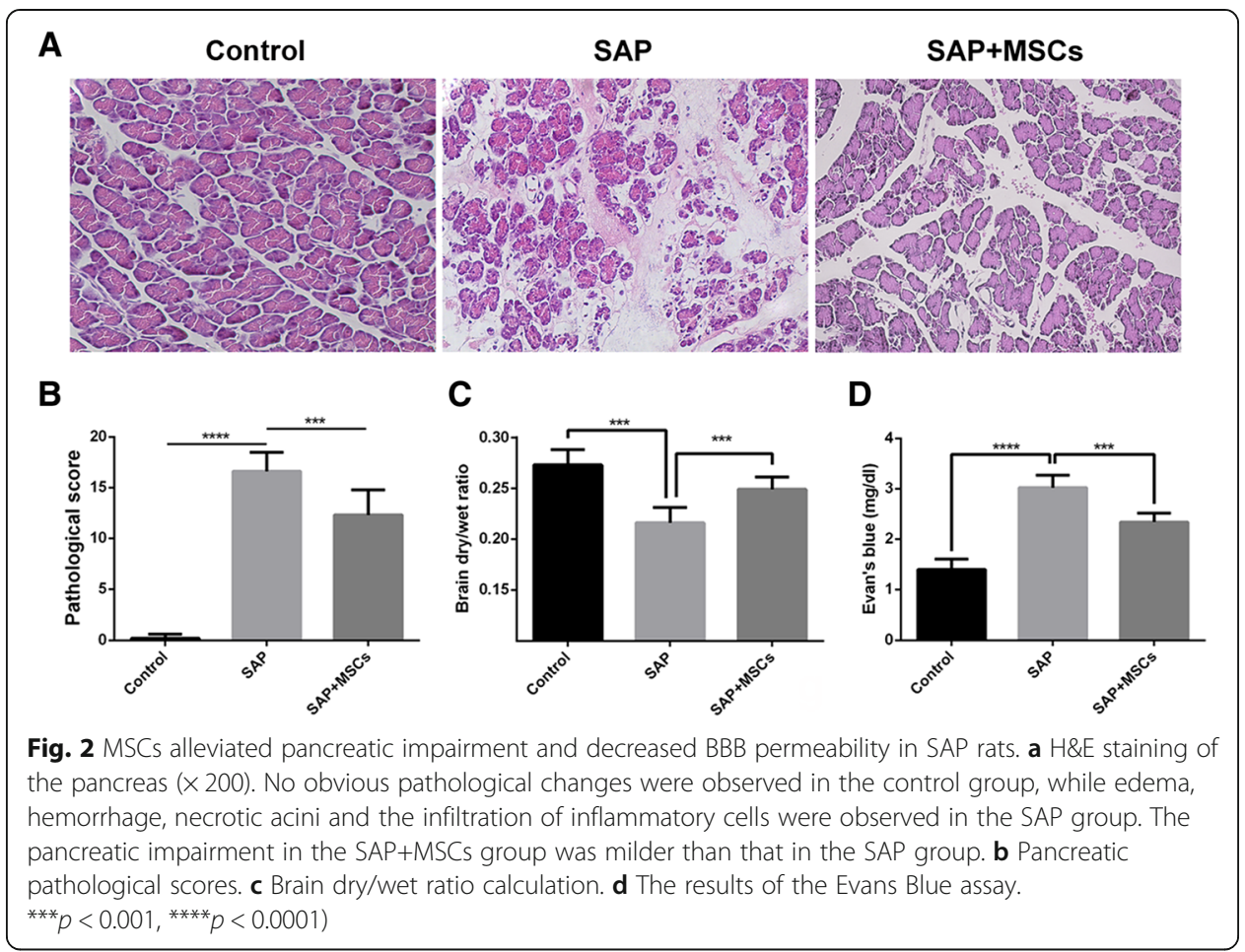

The pancreatic impairment in the SAP+MSCs group was milder than in the SAP group. In concordance with these changes, the pancreatic pathological scores also showed that the SAP group had a significantly higher score than the control group, and that the score decreased with MSC treatment ( $p<0.001$; Fig. 2b).

To assess the potential impacts of MSCs on the BBB in SAP rats, the Evans blue assay and the brain dry/wet ratio were used to evaluate $\mathrm{BBB}$ permeability and secondary cerebral edema. The values of the Evans blue assay for the control, SAP and SAP+MSCs groups were respectively $1.39 \pm 0.21,3.02 \pm 0.24$ and $2.34 \pm 0.18$ $\mathrm{mg} / \mathrm{dl}$ and those for the brain dry/wet ratio were respectively $0.27 \pm 0.01,0.21 \pm$ 0.01 and $0.25 \pm 0.01$ (Fig. 2c and d). The results showed a higher Evans blue value and a lower brain dry/wet ratio value in the SAP group than in the control group, indicating increased $\mathrm{BBB}$ permeability with serious secondary cerebral edema in SAP rats $(p<0.001)$. The changes were partly reversed in SAP rats that received MSC treatment, which showed that MSCs decreased BBB permeability and exhibited protective abilities $(\mathrm{p}<0.001)$.

\section{MSCs attenuated the severity of systematic inflammation in SAP rats}

In the control, SAP and SAP+MSCs groups, the levels of serum amylase were respectively $869 \pm 154,7393 \pm 1071$ and $5042 \pm 1119 \mathrm{IU} / \mathrm{l}$; the levels of serum TNF- $\alpha$ were respectively $82.5 \pm 13.8,237.6 \pm 41.7$ and $147.7 \pm 39.2 \mathrm{pg} / \mathrm{ml}$; and the levels of serum IL-10 were respectively $37.4 \pm 11.8,26.3 \pm 5.6$ and $62.7 \pm 16.6 \mathrm{pg} / \mathrm{ml}$ (Fig. 3ac). The serum levels of amylase and TNF- $\alpha$ were higher and the level of IL-10 was lower in the SAP group than those in the control group $(p<0.05)$. In contrast, the 


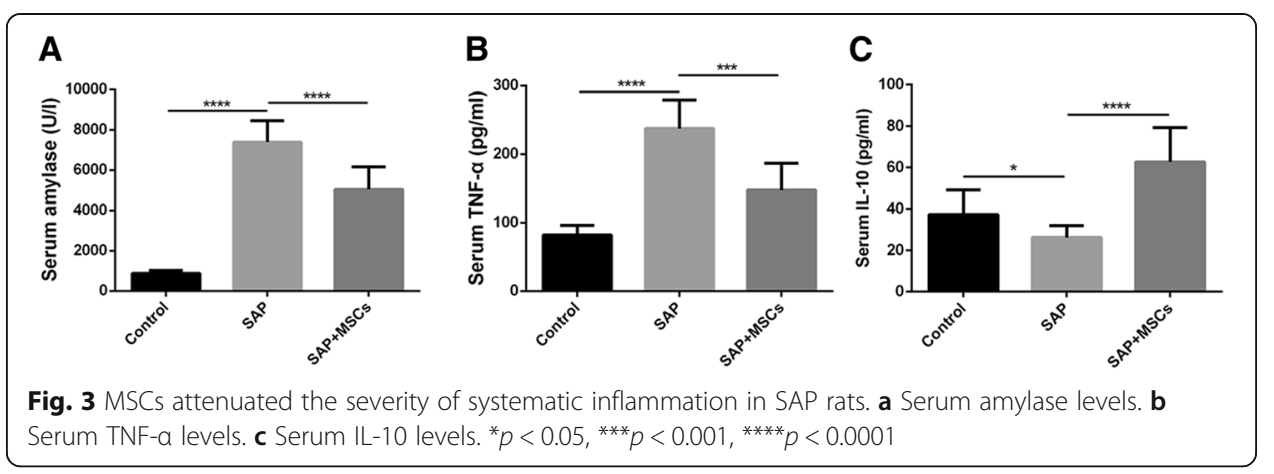

serum levels of amylase and TNF- $\alpha$ were lower and the level of IL-10 was higher in the SAP+MSCs group $(\mathrm{p}<0.001)$ than those in the SAP group. These results show that MSCs attenuate pancreatic impairment and decrease the levels of serum amylase and TNF- $\alpha$ while increasing the level of serum IL-10 in SAP rats.

\section{MSCs decreased the downregulation of claudin-5 in the brains of SAP rats}

The expression level of the tight junction protein claudin- 5 was measured to evaluate for the mechanism leading to the impairment of the BBB. Immunohistochemistry staining showed a higher expression of claudin-5 in BMECs in the control group than in the SAP group. The expression of claudin-5 in the SAP+MSCs group was higher than that in the SAP group, although it was lower than that in the control group (Fig. 4a).

The results of the quantitative real-time PCR and western blotting assays were in accordance with those of the immunohistochemistry staining (Fig. $4 \mathrm{~b}$ and c). These results revealed that claudin- 5 was downregulated in the brains of SAP rats compared to its expression in the controls. This might have contributed to the increased BBB permeability. Treatment with MSCs partly reversed the downregulation of claudin-5, maintaining the stability of the BBB.

\section{MSCs reduced BMEC apoptosis in the brains of SAP rats}

TUNEL staining was performed to assess apoptosis in the brains of SAP rats. TUNEL staining revealed no apoptosis in the control group, but numerous apoptotic cells, mainly BMECs, were observed in the SAP group $(p<0.001)$. Fewer apoptotic cells were observed in the SAP+MSCs group than in the SAP group ( $<<0.001$; Fig. 5). The results show that brain cells become apoptotic in SAP and that treatment with MSCs attenuates apoptosis.

\section{MSCs upregulated Bcl-2 expression and downregulated Bax expression in the brains of SAP rats}

To explore the potential molecular apoptotic mechanisms, the expression levels of the apoptotic protein Bax and the antiapoptotic protein Bcl-2 were measured. The results of western blotting assays and quantitative real-time PCR revealed that Bax was upregulated and Bcl-2 was downregulated in the SAP group compared with the levels in the control group ( $p<0.05$; Fig. $6 \mathrm{a}$ and $\mathrm{b})$. 


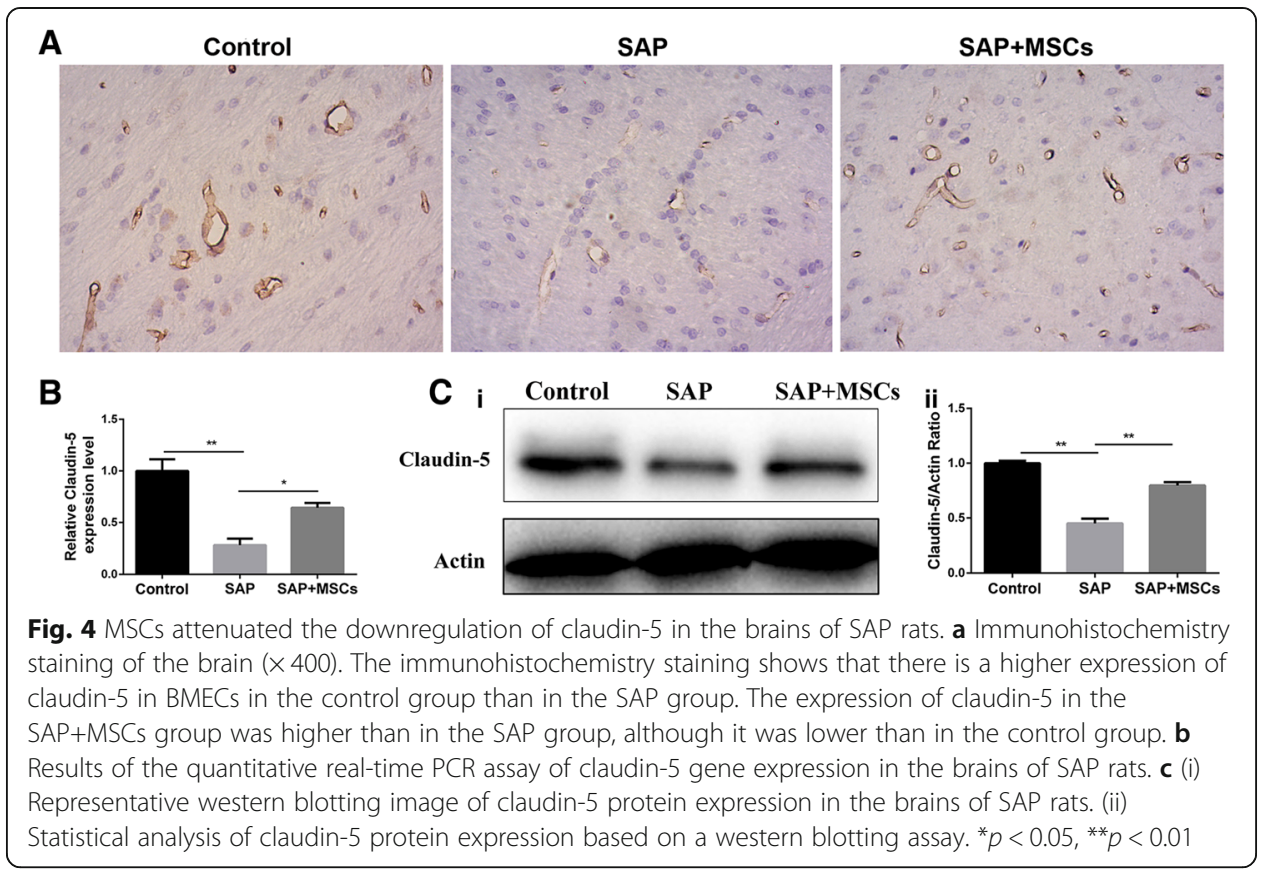

The results of the TUNEL staining show numerous apoptotic cells, and the upregulation of $\mathrm{Bax}$ and downregulation of $\mathrm{Bcl}-2$ are possible stimuli of brain cell apoptosis in the SAP rats. The lower expression level of Bax and higher expression level of Bcl-2 in the SAP+MSCs group than in the SAP group $(\mathrm{p}<0.05)$ indicate that the MSCs partly reversed the modulation of Bax and Bcl-2. In summary, MSCs decreased the upregulation of Bax and the downregulation of $\mathrm{Bcl}-2$ to produce anti-apoptotic effects on brain cells in SAP rats.

\section{MSCs reduced the upregulation of MMP-9 in the brains of SAP rats}

The expression of MMP-9, which has been reported to be a cause of increased BBB permeability, was also measured. A western blotting assay showed that there was a higher expression level of MMP-9 in the SAP group than in the control group $(\mathrm{p}<0.05)$, while the expression level was reduced in the SAP+MSCs group $(\mathrm{p}<0.05)$ (Fig. 7) compared to that in the control group. The results show that MMP-9 was upregulated in the SAP group and that this upregulation was reduced by MSCs.

\section{Discussion}

Severe acute pancreatitis (SAP) is a critical acute abdominal disease characterized by systemic inflammatory response syndrome (SIRS) in the clinical setting [20]. In a cascading reaction, capillary endothelial cells can easily be attacked by a large number of circulating inflammatory cytokines, resulting in capillary leak syndrome (CLS), which is a serious impairment of the endothelial barrier [21, 22].

The blood-brain barrier (BBB) protects the CNS from pathogens. It is an ideal endothelial barrier with very strong barrier properties [23, 24]. Impairment of the BBB refers to CLS in the brain. In SAP, it is associated with increased permeability. The treatment 


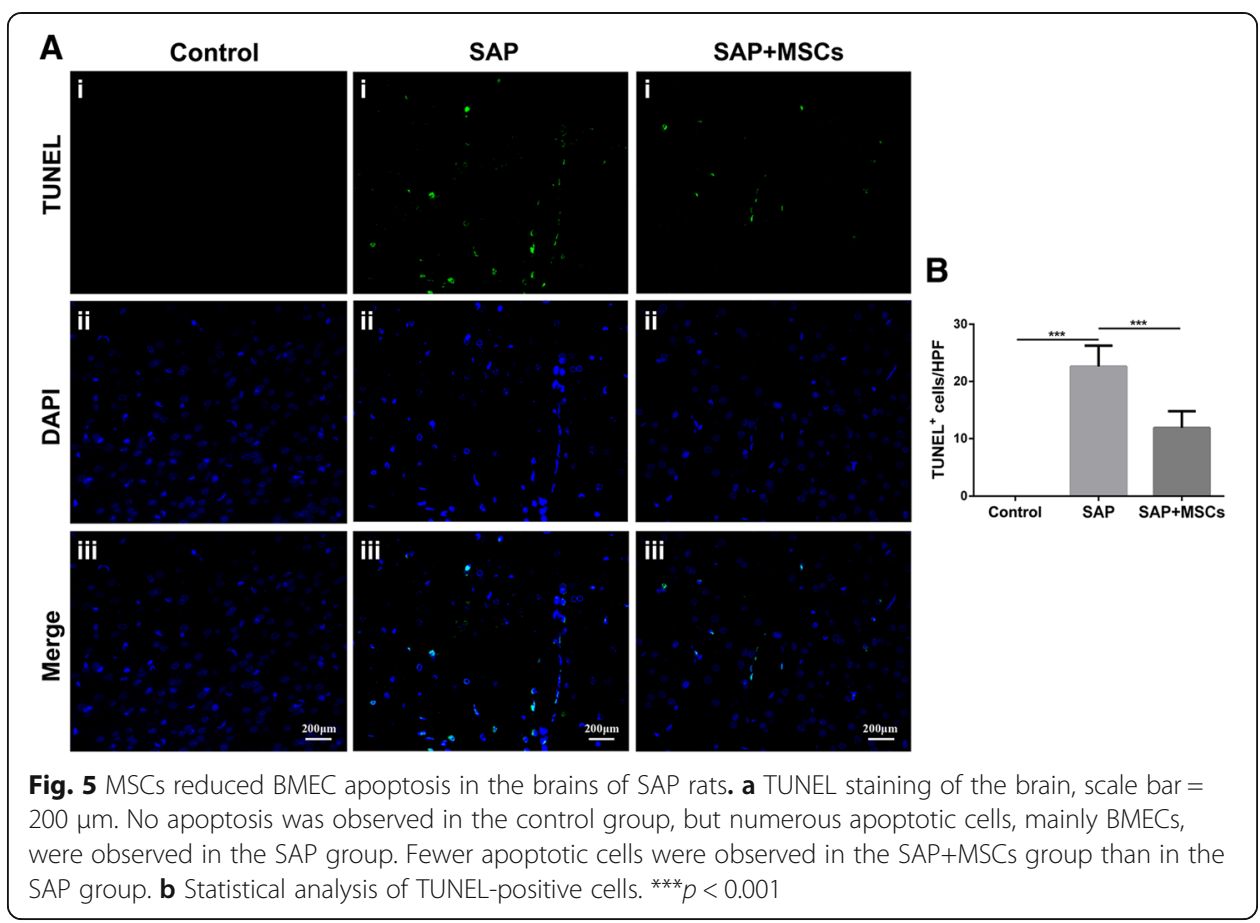

of CLS is a critical component of SAP therapy, and the prevention and treatment of pancreatic encephalopathy (PE) mainly lie in the protection of the BBB. It is essential for reducing complications and decreasing the mortality rate.

Lesions of any component can impair the BBB, meaning a decrease in integrity and function [25] and an increase in permeability. Our study used the Evans Blue assay and the brain dry/wet ratio calculation to confirm the impairment of the BBB in SAP rats with increased permeability and secondary cerebral edema, which is in line with results of a previous study [26].

We also explored the underlying mechanisms of the impairment of the BBB and came to the following conclusions. First, injury or apoptosis of BMECs could cause BBB impairment. The BMECs are directly exposed to the circulating blood flow and could be easily affected by inflammatory cytokines in response to injury or apoptosis during inflammatory situations [27]. In SAP, the remarkably increased levels of serum amylase and inflammatory cytokines, such as TNF- $\alpha$, might lead to BMEC injury. TUNEL staining revealed numerous apoptotic BMECs in SAP rats, which was in line with previously published reports. In combination with the results of quantitative real-time PCR and western blotting assays, which showed a remarkable increase in Bax expression and a decrease in Bcl-2 expression, we infer that the upregulation of Bax and downregulation of Bcl-2 is a possible mechanism of BMEC apoptosis.

Second, the downregulation of the tight junction protein claudin- 5 could contribute to $\mathrm{BBB}$ impairment. Claudins are important components that maintain the tight junctions that are responsible for BBB integrity $[28,29]$. Claudin-5 is the main transmembrane protein component of tight junctions in BMECs, and it has been reported that claudin-5 is the only molecule whose function is specifically required to maintain the BBB [7]. Previous studies have demonstrated that paracellular tight junctions are 


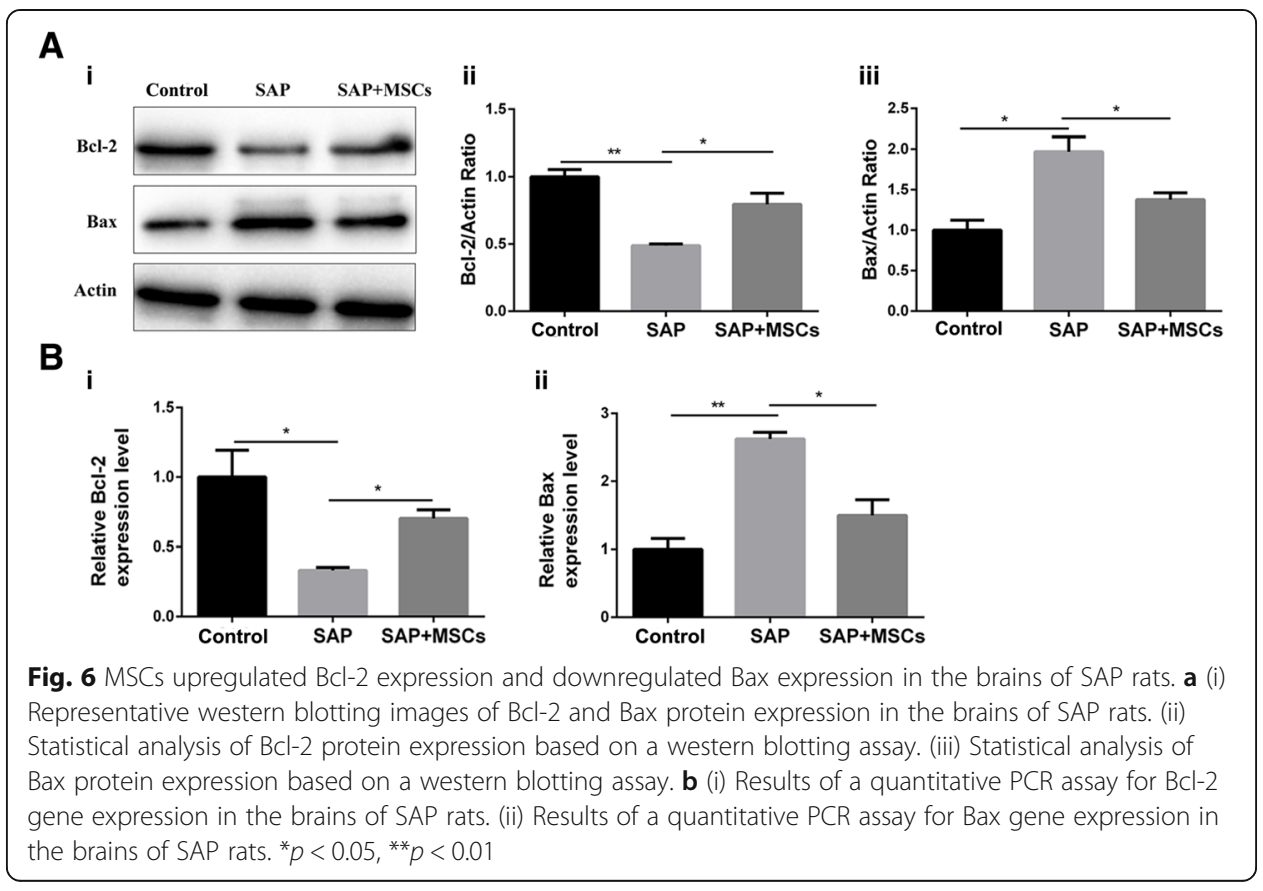

damaged [30] and claudin-5 expression was downregulated [31, 32] under inflammatory conditions in vitro and in vivo, resulting in increased BBB permeability. In this study, the results of our immunohistochemistry, quantitative real-time PCR and western blotting assays show a significant decrease in claudin-5 expression in the brains of SAP rats compared to that in the controls. These results are in accordance with previous studies that indicated that the downregulation of claudin- 5 might contribute to the loss of integrity of the BBB.

Third, the upregulation of MMP-9 expression may contribute to BBB impairment. The expression of MMP-9 is usually upregulated in inflammatory conditions or in ischemia-hypoxia situations. It has been reported that MMP-9 induces the structural disruption of paracellular tight junctions, leading to BBB impairment in vitro [33]. Studies also showed that MMP-9 induces the degradation of collagen component $[34,35]$ and the downregulation of tight junction proteins [8], with increased BBB permeability under inflammatory conditions in vivo. Interestingly, our western blotting results show a noticeably increased expression level of MMP-9 in the brains of SAP rats. Thus, the upregulation of MMP-9 expression is also an important cause of the impairment of the BBB in SAP.

MSCs have been widely studied in many areas because of their pleiotropic abilities, such as multipotential differentiation, low immunogenicity and paracrine function. It has been reported that MSCs can migrate to damaged tissue and secrete trophic factors [36, 37], including cytokines and growth factors, or differentiate into functional local cells to promote tissue repair. MSCs have also been investigated for immunomodulatory abilities resulting from their secretion of various anti-inflammatory molecules [38]. MSC transplantation has been studied in several digestive diseases, in both animal models [39] and clinical trials [40]. Many studies have investigated the therapeutic effects of MSCs on acute pancreatitis 


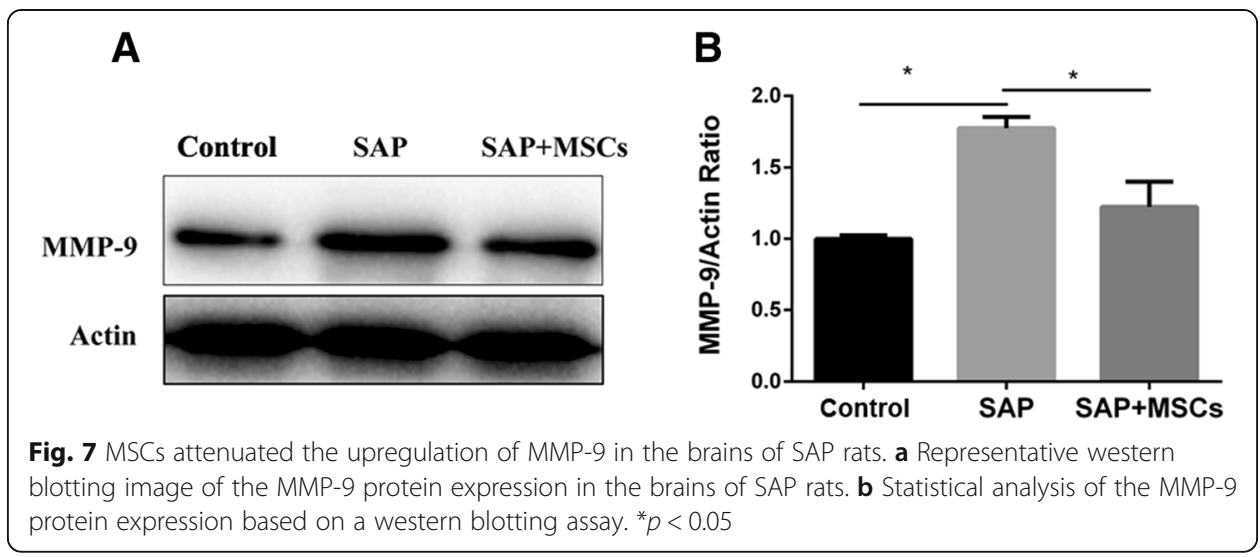

[41-43], but only a few studies have focused on brain damage and investigated the underlying mechanisms.

In this study, we found that MSCs protected the BBB, decreasing its permeability in SAP rats. We explored the underlying mechanisms of MSC protective effects on the $\mathrm{BBB}$ and came to the following conclusions.

First, the use of MSCs in SAP rats decreases the serum levels of amylase and TNF- $\alpha$ and increases the serum level of IL-10, thereby alleviating pancreatic impairment. The serum amylase and TNF- $\alpha$ levels decreased after MSC treatment in this study, which was in accordance with the results of previous studies [11]. Decreased amylase and TNF- $\alpha$ levels attenuate the severity of the systemic inflammation, pancreatic impairment and the injury or apoptosis of BMECs. IL-10 is a wellknown anti-inflammatory cytokine with strong immunomodulatory and antiapoptotic abilities.

Second, MSCs reduce BMEC apoptosis levels. TUNEL staining showed that there were fewer apoptotic BMECs in SAP rats after MSC treatment. This result might mainly be attributed to the decreased serum levels of inflammatory cytokines, including TNF- $\alpha$, and the increased serum IL-10 level, which was in line with a previous study [44]. It has been reported that IL-10 has anti-apoptotic effects on endothelial cells during inflammatory situations [45]. Consequently, the increased serum IL-10 levels might contribute to the anti-apoptotic effects of MSCs. In our study, the expression of Bax decreased and the expression of $\mathrm{Bcl}-2$ increased in the brains of SAP rats that were treated with MSCs, indicating that MSCs have antiapoptotic effects in SAP.

Third, MSCs increase the expression level of claudin-5 in SAP rats. The results of immunohistochemistry, quantitative real-time PCR and western blotting assays show that MSCs increases claudin-5 expression in the brains of SAP rats, and supports the maintenance of BBB integrity. This result might be mainly attributed to the reduced apoptosis of BMECs in SAP rats treated with MSCs, resulting in the increased transcription and translation of claudin-5.

Fourth, MSCs decrease the expression level of MMP-9 in SAP rats. The upregulation of MMP-9 expression is associated with increased BBB permeability, as mentioned above. The results of western blotting assays showed that the expression of MMP-9 was downregulated by MSCs in SAP rats. This result may be due to an MSC-mediated decrease in the serum levels of inflammatory cytokines because the transcription and 
translation of MMP-9 are often initiated by inflammatory cytokines during inflammatory situations [46].

The method of MSCs infusion, including intravenous and intraarterial infusion might exert different effects on the BBB in SAP rats. Intraarterial infusion of MSCs increases the local presence of MSCs in cerebral circulation, but is more difficult than intravenous infusion and has higher risks of microvascular embolization. However, MSCs cannot migrate through the $\mathrm{BBB}$ due to their large size and the barrier properties. Therefore, the protective effects of MSCs on the BBB in SAP rats might be mainly attributed to the immunomodulatory and paracrine functions of MSCs. Notably, MSCderived exosomes are also currently a research focus in cell-free regenerative medicine due to them having similar biological effects but without the ethical issues of cell transplantation. Additional research that further explores the underlying molecular mechanisms will be required in the future.

\title{
Conclusions
}

We found that in SAP rats, increased levels of amylase and inflammatory cytokines, BMEC apoptosis, downregulation of claudin-5 and upregulation of MMP-9 might be the main mechanisms driving the impairment of the BBB, including increased permeability. However, MSCs attenuated the severity of systematic inflammation and pancreatic impairment, reduced BMEC apoptosis, upregulated claudin-5 and downregulated MMP-9, decreasing BBB permeability in SAP rats.

\section{Additional file}

Additional file 1: Table of primers. (DOCX $15 \mathrm{~kb}$ )

\begin{abstract}
Abbreviations
Bax: BCl-2-associated X; BBB: Blood-brain barrier; BCl-2: B-cell lymphoma 2; BMEC: Brain microvascular endothelial cell; CLS: Capillary leak syndrome; DMEM: Dulbecco's modified Eagle's medium; ELISA: Enzyme-linked immunosorbent assay; FBS: Fetal bovine serum; GAPDH: Glyceraldehyde-3-phosphate dehydrogenase; H\&E: Hematoxylin and eosin; IL10: Interleukin-10; MMP-9: Matrix metalloproteinase-9; MSC: Mesenchymal stem cell; PBS: Phosphate-buffered saline; PE: Pancreatic encephalopathy; SAP: Severe acute pancreatitis; SD: Sprague-Dawley; SIRS: Systemic inflammatory response syndrome; TNF-a: Tumor necrosis factor-a; TUNEL: Terminal-deoxynucleotidyl transferase-mediated nick end labelling
\end{abstract}

\section{Acknowledgements}

The authors would like to thank Naishun Liao and Youshi Zheng for their technical support.

\section{Authors' contributions}

$\mathrm{RL}, \mathrm{ML}$ and $\mathrm{HH}$ conceived and designed the experiments, and checked and revised the whole manuscript. RL, ML, ML, $\Pi$ and YP performed the experiments. $R L$ and $M L$ analyzed the data. RL wrote the manuscript. All authors read and approved the final manuscript.

\section{Funding}

The study was funded by the Natural Science Foundation of Fujian Province (No. 2016 J01552), the Medical Center of Minimally Invasive Technology of Fujian Province (No. 171, 2017 and No. 4, 2017), and the Joint Funds of Scientific and Technological Innovation Program of Fujian Province [No. 2017Y9059].

\section{Availability of data and materials}

The data sets supporting the results of this article are included within the article.

Ethics approval

All animal experimental protocols were approved by the Experimental Animals Committee of Fujian Medical University (No. FMU-2017-26). 


\section{Competing interests}

The authors declare that they have no conflict of interest.

\section{Author details}

'Department of General surgery, Fujian Medical University Union Hospital, 29 Xinquan Road, Fuzhou, Fujian 350001, People's Republic of China. ${ }^{2}$ Department of Histology and Embryology, Hunan University of Medicine, Huaihua, Hunan, China. ${ }^{3}$ Department of Orthopedics, Fujian Medical University Union Hospital, Fuzhou, Fujian, China.

Received: 25 January 2019 Accepted: 4 June 2019

Published online: 17 June 2019

\section{References}

1. Jha RK, Ma Q, Sha H, Palikhe M. Protective effect of resveratrol in severe acute pancreatitis-induced brain injury. Pancreas. 2009;38(8):947-53.

2. Keaney J, Campbell M. The dynamic blood-brain barrier. FEBS J. 2015;282(21):4067-79.

3. Zhao Z, Nelson AR, Betsholtz C, Zlokovic BV. Establishment and dysfunction of the blood-brain barrier. Cell. 2015;163(5): 1064-78.

4. Dejana E, Orsenigo F. Endothelial adherens junctions at a glance. J Cell Sci. 2013;126(Pt 12:2545-9.

5. Bazzoni G, Dejana E. Endothelial cell-to-cell junctions: molecular organization and role in vascular homeostasis. Physiol Rev. 2004;84(3):869-901.

6. Le Guelte A, Gavard J. Role of endothelial cell-cell junctions in endothelial permeability. Methods Mol Biol. 2011;763: 265-79.

7. Haseloff RF, Dithmer S, Winkler L, Wolburg H, Blasig IE. Transmembrane proteins of the tight junctions at the blood-brain barrier: structural and functional aspects. Semin Cell Dev Biol. 2015;38:16-25.

8. Kumari R, Willing LB, Patel SD, Baskerville KA, Simpson IA. Increased cerebral matrix metalloprotease-9 activity is associated with compromised recovery in the diabetic db/db mouse following a stroke. J Neurochem. 2011;119(5): 1029-40.

9. Shin TH, Kim HS, Choi SW, Kang KS. Mesenchymal stem cell therapy for inflammatory skin diseases: clinical potential and mode of action. Int J Mol Sci. 2017;18(2):E244.

10. Ding DC, Chang YH, Shyu WC, Lin SZ. Human umbilical cord mesenchymal stem cells: a new era for stem cell therapy. Cell Transplant. 2015;24(3):339-47.

11. Chen Z, Lu F, Fang H, Huang H. Effect of mesenchymal stem cells on renal injury in rats with severe acute pancreatitis. Exp Biol Med (Maywood). 2013;238(6):687-95.

12. Lu F, Wang F, Chen Z, Huang H. Effect of mesenchymal stem cells on small intestinal injury in a rat model of acute necrotizing pancreatitis. Stem Cell Res Ther. 2017:8(1):12.

13. National Research Council (US) Institute for Laboratory Animal Research. Guide for the care and use of laboratory animals. Washington (DC): National Academies Press (US); 1996.

14. Jin M, Chen Y, Zhou Y, Mei Y, Liu W, Pan C, et al. Transplantation of bone marrow-derived mesenchymal stem cells expressing elastin alleviates pelvic floor dysfunction. Stem Cell Res Ther. 2016;7(1):51.

15. Schmidt J, Rattner DW, Lewandrowski K, Compton CC, Mandavilli U, Knoefel WT, et al. A better model of acute pancreatitis for evaluating therapy. Ann Surg. 1992;215(1):44-56.

16. Perides G, van Acker GJ, Laukkarinen JM, Steer ML. Experimental acute biliary pancreatitis induced by retrograde infusion of bile acids into the mouse pancreatic duct. Nat Protoc. 2010;5(2):335-41.

17. Zhou X, Xue C. Ghrelin inhibits the development of acute pancreatitis and nuclear factor kappaB activation in pancreas and liver. Pancreas. 2009;38(7):752-7.

18. Gu C, Wang Y, Li J, Chen J, Yan F, Wu C, et al. Rosiglitazone attenuates early brain injury after experimental subarachnoid hemorrhage in rats. Brain Res. 2015;1624:199-207.

19. Wu C, Hu Q, Chen J, Yan F, Li J, Wang L, et al. Inhibiting HIF-1alpha by 2 ME2 ameliorates early brain injury after experimental subarachnoid hemorrhage in rats. Biochem Biophys Res Commun. 2013;437(3):469-74.

20. Gunjaca I, Zunic J, Gunjaca M, Kovac Z. Circulating cytokine levels in acute pancreatitis-model of SIRS/CARS can help in the clinical assessment of disease severity. Inflammation. 2012;35(2):758-63.

21. Gao SL, Zhang Y, Zhang SY, Liang ZY, Yu WQ, Liang TB. The hydrocortisone protection of glycocalyx on the intestinal capillary endothelium during severe acute pancreatitis. Shock. 2015;43(5):512-7.

22. Bannerman DD, Goldblum SE. Mechanisms of bacterial lipopolysaccharide-induced endothelial apoptosis. Am J Physiol Lung Cell Mol Physiol. 2003;284(6):L899-914.

23. Podjaski C, Alvarez Jl, Bourbonniere L, Larouche S, Terouz S, Bin JM, et al. Netrin 1 regulates blood-brain barrier function and neuroinflammation. Brain. 2015;138(Pt 6:1598-612.

24. Kassner A, Merali Z. Assessment of blood-brain barrier disruption in stroke. Stroke. 2015;46(11):3310-5.

25. Abbott NJ, Patabendige AA, Dolman DE, Yusof SR, Begley DJ. Structure and function of the blood-brain barrier. Neurobiol Dis. 2010;37(1):13-25.

26. Ding Z, Liu J, Lin R, Hou XH. Experimental pancreatitis results in increased blood-brain barrier permeability in rats: a potential role of MCP-1. J Dig Dis. 2012;13(3):179-85.

27. Lin YL, Chang HC, Chen TL, Chang JH, Chiu WT, Lin JW, et al. Resveratrol protects against oxidized LDL-induced breakage of the blood-brain barrier by lessening disruption of tight junctions and apoptotic insults to mouse cerebrovascular endothelial cells. J Nutr. 2010;140(12):2187-92.

28. Kondo N, Ogawa M, Wada H, Nishikawa S. Thrombin induces rapid disassembly of claudin-5 from the tight junction of endothelial cells. Exp Cell Res. 2009;315(17):2879-87.

29. Nitta T, Hata M, Gotoh S, Seo Y, Sasaki H, Hashimoto N, et al. Size-selective loosening of the blood-brain barrier in claudin-5-deficient mice. J Cell Biol. 2003;161(3):653-60.

30. Clark PR, Kim RK, Pober JS, Kluger MS. Tumor necrosis factor disrupts claudin-5 endothelial tight junction barriers in two distinct NF-kappaB-dependent phases. PLoS One. 2015;10(3):e0120075. 
31. Ni C, Wang C, Zhang J, Qu L, Liu X, Lu Y, et al. Interferon-gamma safeguards blood-brain barrier during experimental autoimmune encephalomyelitis. Am J Pathol. 2014;184(12):3308-20.

32. Liu WY, Wang ZB, Wang Y, Tong $L C, L i$ Y, Wei $X$, et al. Increasing the permeability of the blood-brain barrier in three different models in vivo. CNS Neurosci Ther. 2015;21(7):568-74.

33. Wiggins-Dohlvik K, Merriman M, Shaji CA, Alluri H, Grimsley M, Davis ML, et al. Tumor necrosis factor-alpha disruption of brain endothelial cell barrier is mediated through matrix metalloproteinase-9. Am J Surg. 2014; 208(6):954-60 discussion 60.

34. Asahi M, Wang X, Mori T, Sumii T, Jung JC, Moskowitz MA, et al. Effects of matrix metalloproteinase-9 gene knock-out on the proteolysis of blood-brain barrier and white matter components after cerebral ischemia. J Neurosci. 2001;21(19): 7724-32.

35. Vandooren J, Van den Steen PE, Opdenakker G. Biochemistry and molecular biology of gelatinase B or matrix metalloproteinase-9 (MMP-9): the next decade. Crit Rev Biochem Mol Biol. 2013;48(3):222-72.

36. Neuss S, Becher E, Woltje M, Tietze L, Jahnen-Dechent W. Functional expression of HGF and HGF receptor/c-met in adult human mesenchymal stem cells suggests a role in cell mobilization, tissue repair, and wound healing. Stem Cells. 2004;22(3):405-14.

37. Kuan WL, Barker RA. New therapeutic approaches to Parkinson's disease including neural transplants. Neurorehabil Neural Repair. 2005:19(3):155-81.

38. Uccelli A, Moretta L, Pistoia V. Mesenchymal stem cells in health and disease. Nat Rev Immunol. 2008;8(9):726-36.

39. Onishi R, Ohnishi S, Higashi R, Watari M, Yamahara K, Okubo N, et al. Human amnion-derived mesenchymal stem cell transplantation ameliorates dextran sulfate sodium-induced severe colitis in rats. Cell Transplant. 2015;24(12):2601-14.

40. Trounson A, McDonald C. Stem cell therapies in clinical trials: Progress and challenges. Cell Stem Cell. 2015;17(1):11-22

41. Jung KH, Song SU, Yi T, Jeon MS, Hong SW, Zheng HM, et al. Human bone marrow-derived clonal mesenchymal stem cells inhibit inflammation and reduce acute pancreatitis in rats. Gastroenterology. 2011;140(3):998-1008.

42. Zhao H, He Z, Huang D, Gao J, Gong Y, Wu H, et al. Infusion of bone marrow mesenchymal stem cells attenuates experimental severe acute pancreatitis in rats. Stem Cells Int. 2016;2016:7174319.

43. Qian D, Song G, Ma Z, Wang G, Jin L, Hu M, et al. MicroRNA-9 modified bone marrow-derived mesenchymal stem cells (BMSCS) repair severe acute pancreatitis (SAP) via inducing angiogenesis in rats. Stem Cell Res Ther. 2018;9(1):282.

44. Li Q, Song WJ, Ryu MO, Nam A, An JH, Ahn JO, et al. TSG-6 secreted by human adipose tissue-derived mesenchyma stem cells ameliorates severe acute pancreatitis via ER stress downregulation in mice. Stem Cell Res Ther. 2018;9(1):255.

45. Londono D, Carvajal J, Strle K, Kim KS, Cadavid D. IL-10 prevents apoptosis of brain endothelium during bacteremia. J Immunol. 2011;186(12):7176-86.

46. Svedin P, Hagberg H, Savman K, Zhu C, Mallard C. Matrix metalloproteinase-9 gene knock-out protects the immature brain after cerebral hypoxia-ischemia. J Neurosci. 2007:27(7):1511-8.

\section{Publisher's Note}

Springer Nature remains neutral with regard to jurisdictional claims in published maps and institutional affiliations.

Ready to submit your research? Choose BMC and benefit from:
- fast, convenient online submission
- thorough peer review by experienced researchers in your field
- rapid publication on acceptance
- support for research data, including large and complex data types
- gold Open Access which fosters wider collaboration and increased citations
- maximum visibility for your research: over 100M website views per year
At BMC, research is always in progress.
Learn more biomedcentral.com/submissions

